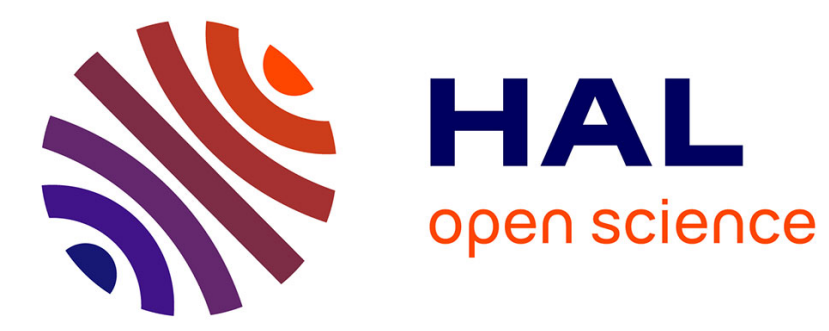

\title{
Molecular simulation of aqueous solutions at clay surfaces
}

Benjamin Rotenberg, Virginie Marry, Natalie Malikova, Pierre Turq

\section{To cite this version:}

Benjamin Rotenberg, Virginie Marry, Natalie Malikova, Pierre Turq. Molecular simulation of aqueous solutions at clay surfaces. Journal of Physics: Condensed Matter, 2010, 22 (28), pp.284114. 10.1088/0953-8984/22/28/284114 . hal-00531718

\section{HAL Id: hal-00531718 \\ https://hal.science/hal-00531718}

Submitted on 9 Nov 2018

HAL is a multi-disciplinary open access archive for the deposit and dissemination of scientific research documents, whether they are published or not. The documents may come from teaching and research institutions in France or abroad, or from public or private research centers.
L'archive ouverte pluridisciplinaire HAL, est destinée au dépôt et à la diffusion de documents scientifiques de niveau recherche, publiés ou non, émanant des établissements d'enseignement et de recherche français ou étrangers, des laboratoires publics ou privés. 


\title{
Molecular simulation of aqueous solutions at clay surfaces
}

\author{
B. Rotenberg ${ }^{1}$, V. Marry ${ }^{1}$, N. Malikova ${ }^{2}$, P. Turq ${ }^{1}$ \\ ${ }^{1}$ UPMC Univ. Paris-06 and CNRS, PECSA, UMR 7195, F-75005, Paris, France \\ ${ }^{2}$ Laboratoire Léon Brillouin, UMR CEA-CNRS 12, CEA Saclay, F-91191, \\ Gif-sur-Yvette, France \\ E-mail: benjamin.rotenberg@upmc.fr
}

\begin{abstract}
We report a molecular simulation study of aqueous solutions at montmorillonite clay surfaces. Unlike most previous studies, we do not focus on the interlayer nanopores, but on both types of external surfaces of clay particles: basal surfaces along the clay layers, and lateral surfaces through which interlayer and larger interparticle pores are linked. We present results on structural, dynamic and thermodynamic properties, such as hydration complexes of ions, H-bonding network, modification of water dynamics with respect to bulk, role of water on cation exchange between interlayer and interparticle pores.
\end{abstract}

PACS numbers: 68.08.-p,66.10.-x,82.30.Rs

Submitted to: J. Phys.: Condens. Matter - Special Issue "Water at interfaces" 


\section{Introduction}

Clays are porous materials found in large quantities at the Earth's surface. Many environmental and industrial processes exploit their mechanical (swelling), catalytic or retention (cationic exchange, anionic exclusion) properties. The possible use of clays as part of a natural or engineered barrier for the geological disposal of toxic and radioactive waste $[1,2]$ has motivated experimental and theoretical studies aiming at elucidating the transport mechanisms of ions, either naturally present in the medium or potential radionuclides, and water through clays. They also play a crucial role as caprocks above natural gas reservoirs or future carbon dioxide repositories.

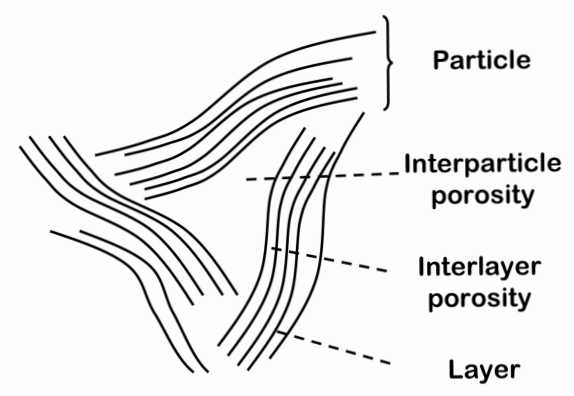

Figure 1. Schematic view of pores in clays. The stacking of clay layers forms particles containing interlayer pores $(\leq \mathrm{nm})$. The aggregation of particles leaves empty regions referred to as interparticle pores (nm to $\mu \mathrm{m}$ ). Interlayers can accomodate a few molecular layers of water, and the interparticle pores can be filled with an electrolyte solution, i.e. water and ions.

Clay minerals are aluminosilicates consisting of negatively charged layers or lamellae. This charge is compensated by solvated cations which can move within the interlayer spacing originating from the stacking of the mineral layers. These stacks form anisotropic particles, with a typical thickness of $\sim 10 \mathrm{~nm}$ and a typical width of a few $\sim$ $100 \mathrm{~nm}$. These particles also aggregate to form larger structures with a corresponding porosity (Fig. 1). The multiscale structure of clays makes it particularly challenging to understand the retention and transport properties. Water plays a crucial role in (1) determining the state of the ions in the medium: are they found in solution in large pores, at the surface of clay particles, or even in particle interlayers? (2) determining the overall transport properties: what are the hydrodynamic boundary conditions at the external surface of clay particles, and can we understand the interplay between the interlayer nanoporosity and interparticle microporosity?

Molecular simulations have proved a powerful tool to understand the structural [3, 4, 5, 6, 7], thermodynamic $[8,9,10,11,12,13]$ and dynamic $[14,15,16,17]$ properties of clay interlayers. Previous studies on basal surfaces of pyrophillite, laponite, montmorillonite and beidellite clays focussed on the properties of ions in their 
vicinity, in particular their possible sorption at the mineral surface $[18,19,20]$. The structure and diffusion of water at the surface of other layered minerals such as brucite, gibbsite, hydrotalcite, muscovite, talc [21, 22, 23, 24] or tobermorite [25] have also been investigated. In this paper, we report molecular simulations of water at external surfaces of clay particles, both at basal surfaces, i.e. along clay layers, and lateral surfaces, where transfers between interlayer and interparticle pores may occur via particle edges. The paper is organized as follows: Section 2 first introduces the simulated systems and the methods used. Then results on basal surfaces are reported in section 3 . Finally, section 4 investigates the exchange of water between interlayer and micropore via lateral surfaces and the role of water in the exchange of cations.

\section{Systems and methods}

\subsection{The clay-water systems}

We consider a smectite clay of the montmorillonite type. Each clay layer consists of one sheet of octahedral aluminum oxide between two sheets of tetrahedral silicon oxides. A fraction of $\mathrm{Al}^{3+}$ are substituted by $\mathrm{Mg}^{2+}$ and the idealized unit cell formula is : $\mathrm{C}_{0.75} \mathrm{Si}_{8}\left(\mathrm{Al}_{3.25} \mathrm{Mg}_{0.75}\right) \mathrm{O}_{20}(\mathrm{OH})_{4}$. The negative charge of $0.75 e$ per unit cell is compensated by 0.75 monovalent counterions $\mathrm{C}^{+}=\mathrm{Na}^{+}$or $\mathrm{Cs}^{+}$. The atomic structure of the clay layer was taken from X-ray diffraction measurements [26, 27]. The layer thickness is $6.54 \AA$. In order to investigate the two types of external surfaces, we have simulated two distinct systems which are illustrated on figure 2 .

For the study of basal surfaces, the horizontal dimensions of the simulation box are $35.88 \times 41.44 \AA^{2}$ corresponding to $4 \times 8$ unit cells. The simulation box contains one layer cut into two halves, and the micropore contains, in addition to the $\mathrm{Na}^{+}$or $\mathrm{Cs}^{+}$counterions, 1600 water molecules and 28 salt pairs $-\mathrm{NaCl}$ or $\mathrm{CsCl}$, depending on the counterion. Prior to molecular dynamics simulations, the distance between layers is equilibrated using Monte-Carlo simulations in the $N \sigma_{z z} T$ ensemble, with a stress $\sigma_{z z}=1$ bar in the direction perpendicular to the layers and a temperature $T=298 \mathrm{~K}$. The equilibrium vertical dimension is $42.90 \AA$ in the sodium case, and the same value is taken for simulations with cesium. The salt concentration in the middle of the pore is $\sim 1$ mol. $\mathrm{dm}^{-3}$.

For the lateral surfaces, the system contains a short clay particle facing a bulk electrolyte solution. Each layer consists of $2 \times 4$ unit cells, with the longest dimension along the $x$-axis, and 4 half-unit cells on each side (along $y$ ) corresponding to [010] edges and whose structure, illustrated in figure 2, was obtained by cutting the unit cell and saturating the broken bonds with $\mathrm{H}$ or $\mathrm{OH}$ groups. The interlayer distance between the layers midplanes was fixed to $d=15.4 \AA$ which corresponds to a water bilayer [28]. The micropore has a width of $30 \AA$, divided in $15 \AA$ on each side of the particle. The overall dimensions of the simulation box are $63.0 \times 20.72 \times 30.8 \AA^{3}$, and periodic boundary conditions in all directions are used. The simulation box contains 

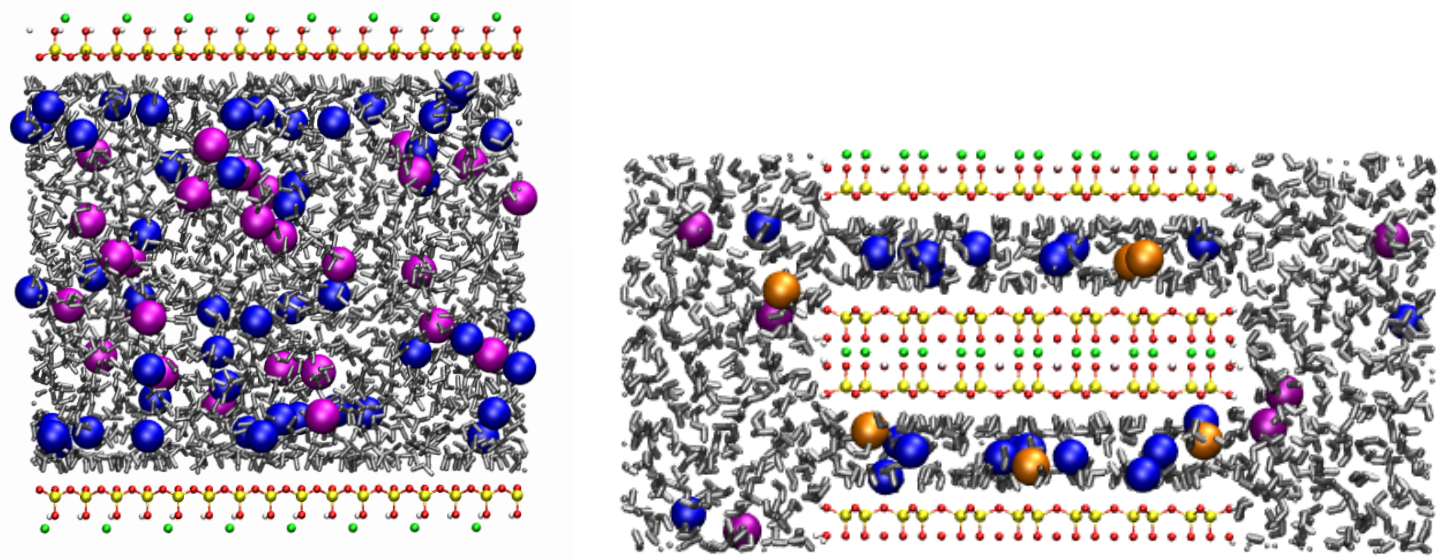

Figure 2. Left: Basal surfaces of clay particles (System I). The mineral surfaces consist of one clay layer of $4 \times 8$ unit cells, divided in two halves. The micropore contains $24 \mathrm{Na}^{+}$or $\mathrm{Cs}^{+}$counterions, 1600 water molecules and $28 \mathrm{NaCl}$ or CsCl salt pairs. Right: Lateral surfaces of clay particles (System II). The clay particle of length $3.3 \mathrm{~nm}$ is in contact with a micropore (1.5 nm on each side of the particle). The interlayer distance corresponds to a water bilayer. Green : $\mathrm{Al}$ and $\mathrm{Mg}$; yellow : $\mathrm{Si}$; red : O; white : H; blue : Na; orange : Cs; pink : $\mathrm{Cl}$; silver : Water molecules. In both cases the $z$ axis corresponds to the direction perpendicular to the basal surfaces. In the second case the $x$ axis refers to the direction perpendicular to the particle/pore interface.

898 water molecules, the interlayer counterions and $6 \mathrm{NaCl}$ pairs in the micropore, corresponding to an electrolyte concentration of $\left.0.52 \mathrm{~mol} . \mathrm{dm}^{-3}\right)$.

\subsection{Simulation details}

The interactions between all atoms were modelled using pairwise potentials:

$$
V_{i j}=\frac{q_{i} q_{j}}{4 \pi \epsilon_{0} r_{i j}}+4 \epsilon_{i j}\left[\left(\frac{\sigma_{i j}}{r_{i j}}\right)^{12}-\left(\frac{\sigma_{i j}}{r_{i j}}\right)^{6}\right]
$$

with $q_{i}$ the partial charge of atom $i$, and the Lennard-Jones (LJ) parameters $\epsilon_{i j}$ and $\sigma_{i j}$ are calculated from individual $\epsilon_{i i}$ and $\sigma_{i i}$ using Lorentz-Berthelot mixing rules [29]. Clay layers are treated as rigid. Water was described with the rigid SPC/E model [30]. The ion LJ parameters are taken from [31] and were shown to correctly describe the diffusion of ions in bulk water. Parameters for bulk clay atoms are taken from Smith [5]. The conjunction of all these parameters was shown to reasonably describe the dynamics of water and ions in clay interlayers $[28,32,17]$. The LJ parameters for edge oxygen atoms were identical to those of the bulk surface oxygen atoms, which are the same as $\mathrm{SPC} / \mathrm{E}$ water oxygen atoms in the Smith force field. The atomic parameters defining the force field are summarized in Table 1. For lateral surfaces, we determined the partial charges of the edge atoms from the Restrained Electrostatic Potential (RESP) method [33, 34], using Density Functional Theory calculations on a short fragment of 
pyrophyllite, an uncharged analogue of montmorillonite. The resulting parameters can be found in Reference [35]. We used a cut-off of $8 \AA$ for short-range interactions and Ewald summation for long-range electrostatic interactions.

\begin{tabular}{|c|c||c|c|c|}
\hline Molecule & Atom & $\mathrm{q}_{i}(\mathrm{e})$ & $\sigma_{i i}(\AA)$ & $\varepsilon_{i i}\left(\mathrm{~kJ} . \mathrm{mol}^{-1}\right)$ \\
\hline \multirow{4}{*}{ Clay } & $\mathrm{Al}$ & 3.0 & 0.0 & 0.0 \\
\cline { 2 - 5 } & $\mathrm{Mg}(\mathrm{Subst.} \mathrm{Al})$ & 2.0 & 0.0 & 0.0 \\
\cline { 2 - 5 } & $\mathrm{Si}$ & 1.2 & 1.84 & 13.18 \\
\cline { 2 - 5 } & $\mathrm{O}($ Surface$)$ & -1.0 & 3.166 & 0.650 \\
\cline { 2 - 5 } & $\mathrm{O}\left(O_{h}\right)$ & -1.424 & 3.166 & 0.650 \\
\cline { 2 - 5 } & $\mathrm{O}\left(T_{d}\right)$ & -0.8 & 3.166 & 0.650 \\
\cline { 2 - 5 } & $\mathrm{H}$ & 0.424 & 0.0 & 0.0 \\
\hline \multirow{3}{*}{ Water } & $\mathrm{O}$ & -0.848 & 3.166 & 0.650 \\
\cline { 2 - 5 } & $\mathrm{H}$ & 0.424 & 0.0 & 0.0 \\
\hline \multirow{3}{*}{ Ions } & $\mathrm{Na}$ & 1.0 & 2.587 & 0.418 \\
\cline { 2 - 5 } & $\mathrm{Cs}$ & 1.0 & 3.883 & 0.418 \\
\cline { 2 - 5 } & $\mathrm{Cl}$ & -1.0 & 4.401 & 0.418 \\
\hline
\end{tabular}

Table 1. Charges $q_{i}$ and atomic Lennard-Jones parameters $\sigma_{i i}$ and $\varepsilon_{i i}$. The pair Lennard-Jones parameters are obtained from the Lorentz-Berthelot mixing rules.

All molecular dynamics simulations in the $N V T$ ensemble were performed using the DLPOLY software package [36]. The temperature was maintained at $298 \mathrm{~K}$ using a Nosé-Hoover thermostat [29] and the equations of motion for the rigid water molecules were integrated using the SHAKE algorithm [37]. We used a timestep of 1 fs. For basal surfaces, density profiles are determined from a 2.5 ns trajectory, while water dynamic properties are computed from 260 ps simulations sampled every 50 fs. For lateral surfaces, two 10 ns trajectories were used in order two ensure proper sampling of the simulation volume by all species.

\section{Water at basal surfaces of clay particles}

\subsection{Structure}

The distributions of water and ions at a basal surface (System I) are reported in figure 3. The water profiles are almost independent of the nature of the counterion, and two to three water layers are observed at the surface. Anions are excluded from the vicinity of the surface due to the negative charge of the mineral. The location of the counterions depends on their nature: $\mathrm{Cs}^{+}$are found closer to the surface than $\mathrm{Na}^{+}$. This observation, consistent with the behaviour of these ions in the interlayer [28], is a consequence of the different types of surface complexes: $\mathrm{Cs}^{+}$form mainly inner-sphere complexes (ISC), with at least one surface oxygen atom in the first coordination shell of the ion, while $\mathrm{Na}^{+}$form mainly outer-sphere complexes (OSC) with only water molecules in the first 
coordination shell. This fact has already been observed in NMR [38] and EXAFS [39] experiments. Note that the Debye screening length of electrostatic interactions is very short $(\sim 1 \mathrm{~nm})$ for this high salt concentration, so that the ionic density profiles reach their bulk value not far from the surface.

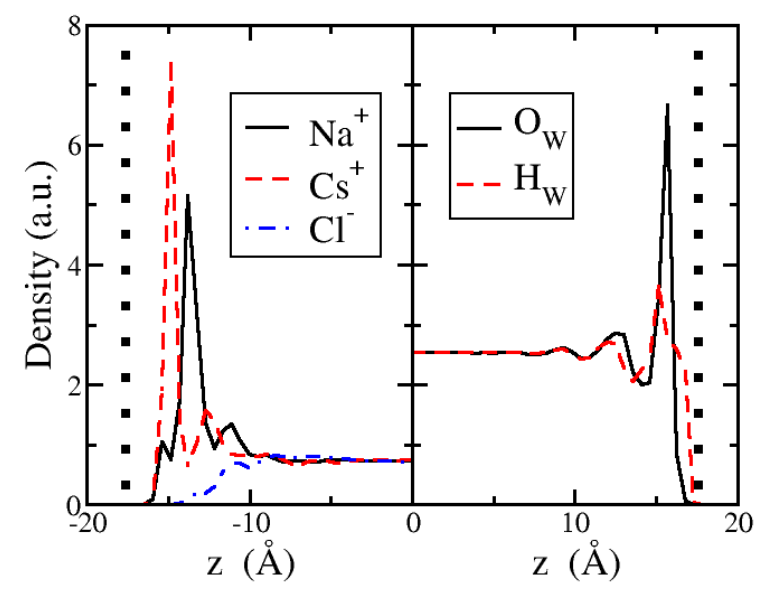

Figure 3. Distribution of ions (left) and water (right) in the micropore facing basal surfaces. The vertical dotted lines indicate the location of the surface oxygen atoms. In the right figure, the density of hydrogen atoms has been scaled by a factor 0.5 . The density profiles are symmetric with respect to the center of the pore, and only one half is reported. Anions are excluded from the surface, and the structure of surface complexes depends on the nature of the counterion: $\mathrm{Cs}^{+}$form mainly inner-sphere complexes and are thus found closer to the surface than $\mathrm{Na}^{+}$, which form mainly outer-sphere complexes. Water is ordered near the surface, over two to three layers. The results for $\mathrm{Na}^{+}$and $\mathrm{Cs}^{+}$are almost identical, and only that for $\mathrm{Na}^{+}$are shown.

In a previous paper [40], we analyzed down to a great detail the structure of the hydrogen bonding network in the surface water layer, defined as the molecules whose oxygen atom is at a distance smaller than $4 \AA$ from the plane of surface oxygen atoms (region "a" in figure 4). We use the following definition of a $\mathrm{H}$-bond [41, 42, 43]: the distance between oxygen atoms must be $d\left(\mathrm{O}_{1} \mathrm{O}_{2}\right)<3.5 \AA$ and the angle $\theta\left(\mathrm{H}_{1} \mathrm{O}_{1} \mathrm{O}_{2}\right)<$ $30^{\circ}$. Approximately $50 \%$ of surface water molecules donate one $\mathrm{H}$-bond to surface oxygen atoms, and these molecules will be referred to as "type A". Approximately $40 \%$ of surface molecules donate at least one H-bond to a type A molecule, without forming a H-bond with the surface. Surface molecules that are not H-bonded to the surface are referred to as "type B".

\subsection{Dynamics}

We now turn to the dynamic properties of water near basal surfaces. Figure 4 reports the water diffusion coefficient $D_{\|}$along the surface as a function of the distance to the surface. The micropore is first divided into four distinct regions: the surface water 


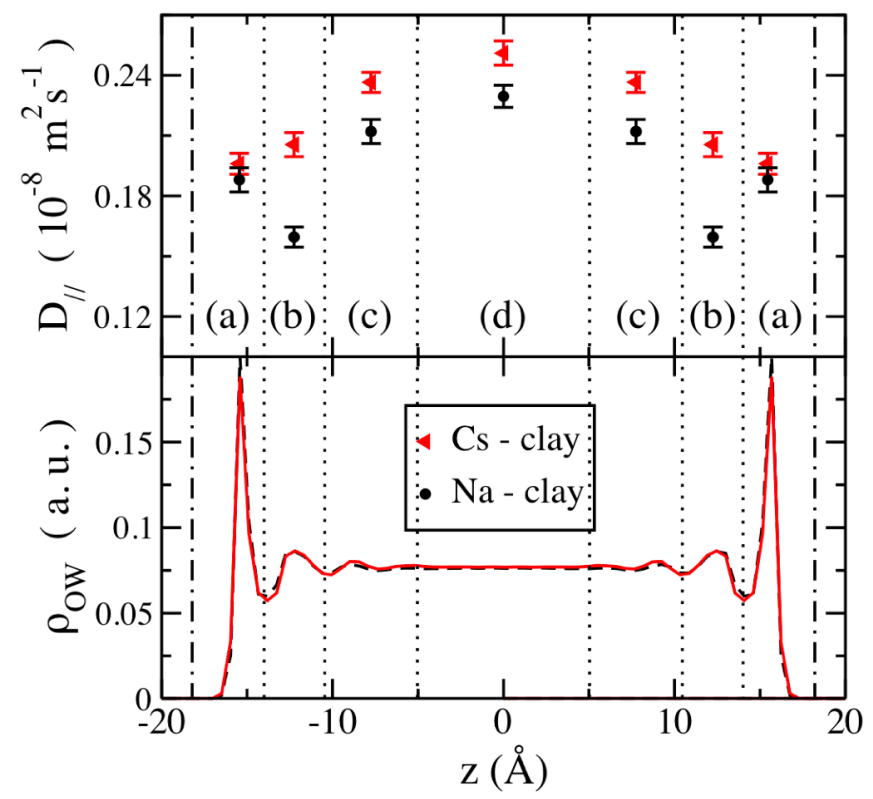

Figure 4. Water diffusion coefficient along the surfaces, as a function of distance to the surface (top), for $\mathrm{Na}^{+}(\bullet)$ and $\mathrm{Cs}^{+}(\triangleleft)$ counterions. Results are computed from the mean-square displacement of water molecules remaining in each of the region defined from the density profiles (bottom part of the figure).

molecules (region "a" mentioned above), the second ("b" between 4 and $7.5 \AA$ from the surface) and third ("c" between 7.5 and $13 \AA$ ) water layers, and the bulk electrolyte solution ("d" further than $13 \AA$ ). Diffusion coefficients are then computed in each region from the mean-square displacement by considering only the molecules that have not left the region between time 0 and time $t$. This allows to rigorously define an effective 2D diffusion coefficient in each layer and to avoid the averaging effect which arises from the excursion of molecules between several layers.

For both counterions, $D_{\|}$is smaller close to the surface than in the bulk. Such a decrease has been observed with several uncharged mineral surfaces [44, 45]. An additional mechanism at play with charged clays is the crowding of the surface due to the presence of counterions, and the fact that surface water molecules spend some time in the coordination shell of the ions which diffuse more slowly than water. The diffusion coefficient value in the central layer is comparable to the bulk result for the SPC/E model and the experimental one, namely $2.4 \pm 0.110^{-9} \mathrm{~m}^{2} \mathrm{~s}^{-1}$ [46] and $2.3 \pm 0.110^{-9} \mathrm{~m}^{2} \mathrm{~s}^{-1}$ [47], respectively. The slight deviation comes from the presence of salt at a relatively high concentration. The effect of the counterion on water diffusion in the region "d" is similar to the one observed experimentally for concentrated alkaline chloride solutions. This effect is however less dramatic than the one observed in the second water layer (region "b"): $D_{\|}$is smaller in the $\mathrm{Na}^{+}$case, even smaller in this layer than in the surface layer "a". This observation can be understood from the structure of the surface complexes: 
The ISC formed by $\mathrm{Cs}^{+}$affects only the first water layer, while the OSC formed by $\mathrm{Na}^{+}$ also hinders the movement of molecules in the second layer.

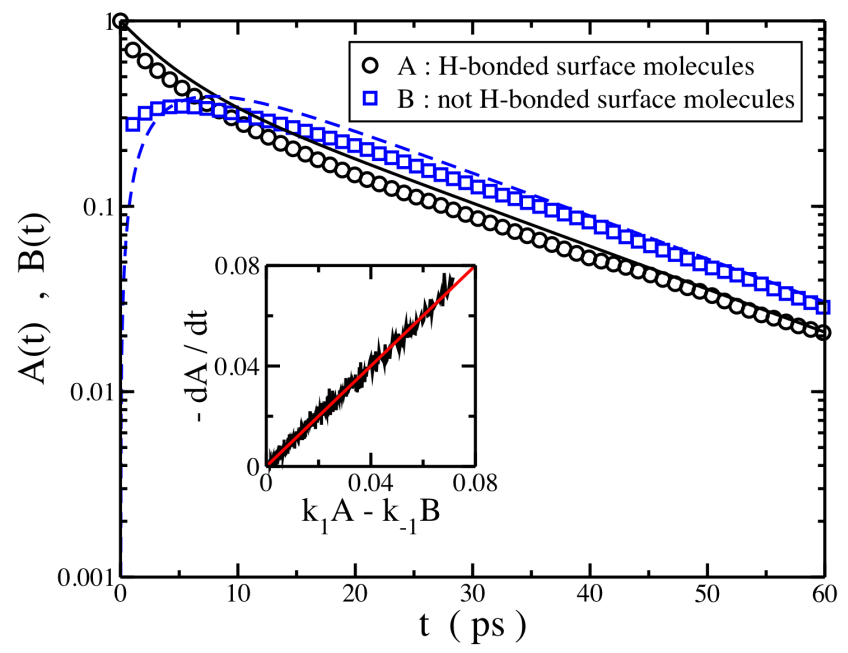

Figure 5. Population of surface water molecules forming (A) and not forming (B) $\mathrm{H}$-bonds with surface oxygen atoms. Only molecules that were initially H-bonded and remained continuously in the surface layer between 0 and $t$ are considered. Simulation results are compared to a simple kinetic model (see text). The inset shows that the assumption of first order kinetics for the breaking and formation of $\mathrm{H}$-bonds is well satisfied for times longer than 2 ps (shorter times are not shown). The semi-logarithmic scale illustrates the exponential decay of both populations at long times.

We have also investigated the dynamics of surface H-bond formation and breaking. The population of surface H-bonded (type A) surface molecules was analyzed by computing the following correlation function [48, 49, 50, 40]:

$$
A(t)=\frac{\langle h(0) h(t) S(t)\rangle}{\langle h(0) S(0)\rangle}
$$

where $h(t)=1$ if the molecule is forming an H-bond with the surface at time $t$, and 0 otherwise, and $S(t)=1$ if the molecule has never left the surface layer between time 0 and time $t$. Since type A molecules are likely to leave the surface layer only after breaking the H-bond with the surface, we introduce, following Luzar and Chandler [41]:

$$
B(t)=\frac{\langle h(0)[1-h(t)] S(t)\rangle}{\langle h(0) S(0)\rangle}
$$

for molecules that were initially surface H-bonded and are still in the surface layer without forming a $\mathrm{H}$-bond with the surface. Both correlation functions are reported in figure 5. They are seen to decay exponentially at long times.

A simple kinetic model can be introduced to interpret the dynamics of $\mathrm{H}$-bonds formation and breaking and the release of molecules from the surface layer. If one assumes first order kinetics for all processes and that only B molecules can leave the 
surface layers, the populations should satisfy:

$$
\frac{\mathrm{d} A}{\mathrm{~d} t}=-k_{1} A+k_{-1} B
$$

with $k_{-1}$ and $k_{1}$ the formation and breaking rates, and

$$
\frac{\mathrm{d} B}{\mathrm{~d} t}=k_{1} A-\left(k_{-1}+k_{0}\right) B
$$

with $k_{0}$ the rate for the release of not $\mathrm{H}$-bonded molecules from the surface layer. The inset of figure 5 shows that equation (4) is well satisfied for times longer than 2 ps, with breaking and formation rates $k_{1}=0.15 \mathrm{ps}^{-1}$ and $k_{-1}=0.07 \mathrm{ps}^{-1}$. Almost the same value is obtain with $\mathrm{Na}^{+}$and $\mathrm{Cs}^{+}$counterions. Short-time deviations from the simple two-state model are also observed in bulk solutions (the notion of surface layer being replaced by that of first neighbour shell) and originate from libration and inter-oxygen vibration at very short time-scales. The rupture and bonding rates are respectively slower and faster than the ones corresponding to H-bonds between water molecules in a bulk salt solution [40]. Two effects might explain these results. On the one hand the negative charge of the mineral tends to orient water molecules in a configuration favourable to the formation of an H-bonds. The importance of this factor could be quantified by comparing the results for an uncharged clay such as pyrophillite. On the other hand the H-bond to the surface may be broken but is reformed with a high probability, due to the decreased number of adjacent water molecules to accept a new H-bond [51].

Figure 5 also shows the evolution of $A$ and $B$ obtained from simulation and within the two-state model for initial conditions $A(0)=1$ and $B(0)=0$. The model semi-

quantitatively reproduces simulation results when the release rate $k_{0} \sim 0.09 \mathrm{ps}^{-1}$ is used. The agreement is very satisfactory, given the simplicity of the kinetic model. This justifies a posteriori the assumption of first order kinetics and the statement that most surface H-bonded molecules break their H-bond with the surface before leaving the surface layer. Note that all processes (formation, rupture, release) occur on the same timescale.

\section{Water and the interlayer-interparticle pore exchange}

We now turn to the exchange of species between interlayer and interparticle pores via lateral surfaces, corresponding to the second system of figure 2.

\subsection{Water exchange via particle edges}

During the $10 \mathrm{~ns}$ simulations, we observe a many exchanges of water molecules between the two types of pores. Within the same time, we observe less exchange events for cations, and no entrance of anions into the interlayer. While the overall interface crossing rates depend on the diffusion coefficient of the species and on the geometry of the system, these observations suggest that the probability for anions reaching the interface from the 
micropore to enter into the interlayer is very small, in agreement with the idea of anion exclusion. On the contrary, water molecules reaching the interface from one or the other direction have a finite probability to cross it. This is confirmed by the relatively flat potential of mean force deduced from the equilibrium density $\mathcal{V}(x)=-k_{B} T \ln \rho_{O W}(x)$, shown in figure 6 . From this free energy profile we identify three distinct regions in the simulation box: interlayer $(|x| \leq 16 \AA)$, micropore $(|x| \geq 23 \AA)$ and interface. The free energy difference between the interlayer and interparticle pore is only of the order of the thermal energy and is easily overcome. The origin of this difference lies in the entropic cost of entering into the particle: the accessible volume per unit length, in the $x$ direction, is $\sim 1 / 3$ of that in the micropore, and the corresponding free energy penalty is $\Delta \mathcal{V} \sim k_{B} T \ln 3=1.1 k_{B} T$. There is no additional barrier at the interface, simply oscillations due to the perturbation of the water structure by the clay edges.

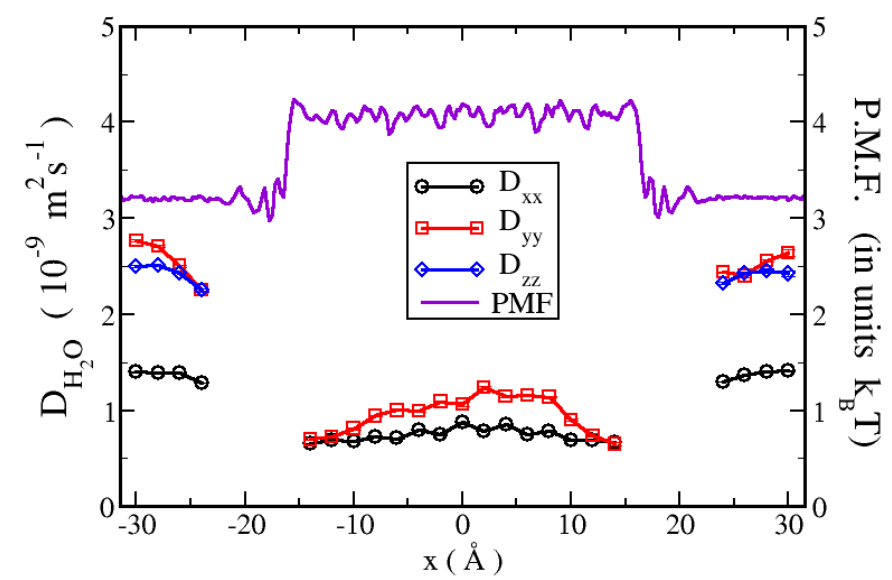

Figure 6. Components of the diffusion tensor of water molecules inside and outside of the clay particle. Also shown is the potential of mean force $\beta \mathcal{V}(x)=-\ln \rho_{O W}(x)$. In the interlayer, there is no diffusion in the direction perpendicular to the surface $\left(D_{z z}=0\right)$ and the two other components are close to the one obtained for infinite clay particles. In the micropore, the components along the particle surface are close to the bulk diffusion coefficient, while the one in the direction perpendicular to the surface is smaller.

In the interlayer and micropore regions, where the potential of mean force is constant, we also computed the diffusion tensor $\operatorname{Diag}\left(D_{x x}, D_{y y}, D_{z z}\right)$ of water molecules. For $D_{x x}(x)$ we used the method of Liu et al. [52, 35]. Figure 6 reports the components of the diffusion tensor of water molecules as a function of position in the simulation box. In the interlayer, the diffusion is two-dimensional ( $D_{z z} \sim 0$, not shown) and almost isotropic in the $x y$-plane $\left(D_{x x} \sim D_{y y}\right) . D_{x x}$ and $D_{y y}$ only slightly depend on position, with a value $\sim 0.9 \pm 0.110^{-9} \mathrm{~m}^{2} \mathrm{~s}^{-1}$. These observations are in agreement with MD simulations of "bulk" interlayers [28, 53] and Quasielastic Neutron Scattering (QENS) experiments [32, 17]. This confirms a posteriori that the small size of the 
simulated clay particle is sufficient to account for the "bulk" clay behaviour. In the micropore, diffusion along the clay particle $\left(D_{y y} \sim D_{z z}\right)$ is similar to bulk water diffusion: The value $2.5 \pm 0.210^{-9} \mathrm{~m}^{2} \mathrm{~s}^{-1}$ is in agreement both with experimental results $\left(2.3 \pm 0.110^{-9} \mathrm{~m}^{2} \mathrm{~s}^{-1}\right)[47]$ and $\mathrm{MD}$ simulations of bulk $\mathrm{SPC} / \mathrm{E}$ water $\left(D^{\mathrm{SPC} / \mathrm{E}}=\right.$ $\left.2.4 \pm 0.110^{-9} \mathrm{~m}^{2} \mathrm{~s}^{-1}\right)[46,54]$. The component normal to the particle surface is smaller than the other two: $D_{x x} \sim 1.5 \pm 0.110^{-9} \mathrm{~m}^{2} \mathrm{~s}^{-1}$. Since it is computed in a region where the density has already reached its bulk value, this means that the effect of the particle/pore interface is longer-ranged on diffusion that on density. A larger simulation box should allow to observe the crossover from the observed $D_{x x}$ values close to the surface to the bulk value at larger distances.

\subsection{The role of water in the exchange of cations}

As mentioned in the introduction, water plays a crucial role in the transport of ions through clays because it determines not only the hydrodynamic properties of fluid flow through the porosity, but also the state of the ions in the material. In particular, it controls the thermodynamics of cation exchange, which is one of the main retention mechanisms of some cations such as $\mathrm{Cs}^{+}$. For the sake of completeness, we report here the insights gained recently on this issue using molecular simulations. As the exchange reaction proceeds, where the aqueous $\mathrm{Cs}^{+}$ion gradually replaces the $\mathrm{Na}^{+}$ions initially in the clay interlayer, while the latter are released into the aqueous phase. While the water content may vary between the initial and final states, it is important to consider mass-balanced reactions, including water, to properly define thermodynamic quantities of reaction. The exchange reaction is thus written:

$$
\overline{\mathrm{Na}^{+}} n \mathrm{H}_{2} \mathrm{O}+\mathrm{Cs}_{(a q)}^{+} \quad \longrightarrow \quad \overline{\mathrm{Cs}^{+}} n \mathrm{H}_{2} \mathrm{O}+\mathrm{Na}_{(a q)}^{+}
$$

with $n$ the number of water per interlayer cation, typically 6-7 and 12-14 for Namontmorillonite in the mono- and bilayer states, respectively. Selectivity measurements have shown that this exchange is thermodynamically favourable. This was usually interpreted as the result of favourable interactions of $\mathrm{Cs}^{+}$with the clay surface. However, Teppen and Miller showed using molecular simulation, that replacing an alkaline ion (e.g. $\left.\mathrm{Na}^{+}\right)$in the interlayer by a larger one $\left(\right.$e.g. $\left.\mathrm{Cs}^{+}\right)$is associated with a large free energy cost, and that the driving force for the exchange is in fact the difference in hydration free energy, i.e. the favourable replacement of $\mathrm{Cs}^{+}$by $\mathrm{Na}^{+}$in the aqueous phase [55]. This finding was later found to explain the observed increase in the selectivity in consolidated clays with mechanical compaction, and thus decreasing water content in the micropores [56].

Using molecular simulations, we recently investigated the role of ion hydration and interlayer water on the exchange enthalpy [57]. The following thermodynamic cycle separates the contribution of the change of ion in both the clay interlayer and the 
aqueous phase :

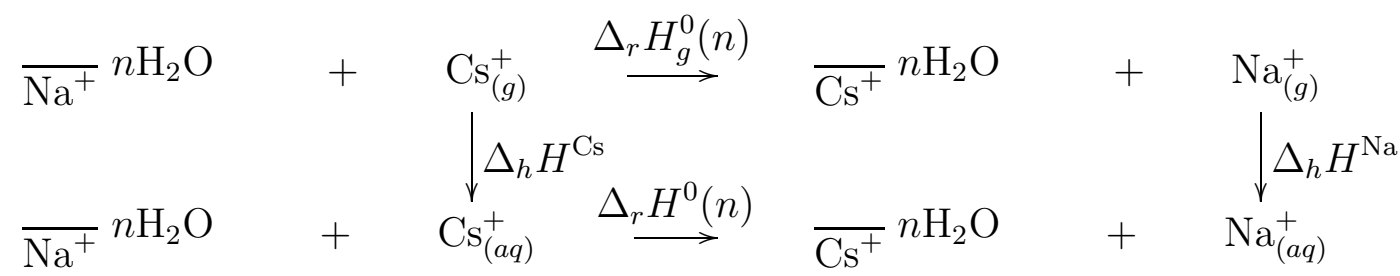

This cycle involves the exchange reaction with ions in the gas phase, where there are no interactions, used as a reference state. The associated thermodynamic quantities are the hydration enthalpy of both cations $\Delta_{h} H^{\mathrm{Na}}$ and $\Delta_{h} H^{\mathrm{Cs}}$, the standard ionic exchange reaction with the gas phase $\Delta_{r} H_{g}^{0}(n)$ and that with the aqueous phase $\Delta_{r} H^{0}(n)$. From this cycle we deduce the relation : $\Delta_{r} H^{0}(n)=\Delta_{r} H_{g}^{0}(n)-\Delta \Delta_{h} H$. We showed that replacing $\mathrm{Na}^{+}$by $\mathrm{Cs}^{+}$in the interlayer is an endothermic process $\left(\Delta_{r} H_{g}^{0}>0\right.$, see Table 2) and that the overall exchange is exothermic $\left(\Delta_{r} H^{0}<0\right)$ only because of the hydration enthalpy difference $\Delta_{h} H=\Delta_{h} H^{\mathrm{Cs}}-\Delta_{h} H^{\mathrm{Na}} \sim 133 \mathrm{~kJ} . \mathrm{mol}^{-1}$ associated with the replacement of $\mathrm{Cs}^{+}$by $\mathrm{Na}^{+}$in the aqueous phase. This conclusion and the evolution with water content were confirmed by microcalorimetry experiments [57].

\begin{tabular}{|c|c|}
\hline$n_{\mathrm{H} 2 \mathrm{O}}$ & $\Delta_{r} H_{g}^{0}\left(\mathrm{~kJ} . \mathrm{mol}^{-1}\right)$ \\
\hline 1 & $+88 \pm 5$ \\
6 & $+101 \pm 3$ \\
12 & $+113 \pm 4$ \\
\hline
\end{tabular}

Table 2. Clay contribution to the standard enthalpy for the exchange reaction between a Na-Clay with $n$ water molecules per cation and a Cs-Clay with $n$ water molecules per cation. The values for $n=1,6$ and 12 correspond to the dry, monolayer and bilayer states, respectively - even if the latter is not experimentally stable in the Cs case.

\section{Conclusion}

We have studied, using molecular dynamics simulations, the structure and dynamics of water at clay surfaces. Unlike most studies, we did not focus on the interlayer nanopores, but on both types of external surfaces of clay particles: basal surfaces along the clay layers, and lateral surfaces through which interlayer and interparticle porosity are linked. Particularly interesting near clay surfaces is the interplay between surface, water and ions. The surface of smectite clays is considered as intrinsically hydrophobic, since uncharged clays do not swell, but substitutions in the mineral layers and the associated counterions, as well as their hydration, critically modify this behaviour. The balance between water/surface, ion/surface and ion/water interactions controls among others the structure of the surface complexes and the thermodynamics of ion exchange. In turn, ions influence the organization and dynamics of water, and one observes specific effects of cations on the properties of surface water. Future work will allow us to consider 
how the properties of the surface water layers affect the hydrodynamic behaviour further away from the surface, and characterize more precisely the electro-osmotic effect at the clay/water interface [58].

\section{Acknowledgments}

This paper is a contribution that concerns recent progresses in the field of computer simulations of water discussed at the CECAM workshop "Modeling and Simulation of Water at Interfaces from Ambient to Supercooled Conditions" supported by ESFSimbioma and CECAM.

[1] ANDRA. Évaluation de la faisabilité du stockage géologique en formation argileuse. Dossier 2005 Argile : Synthèse, Châtenay-Malabry, France, 2005.

[2] M. Bradbury and B. Baeyens. Near Field Sorption Data Bases for Compacted MX-80 Bentonite for Performance Assessment of a High-Level Radioactive Waste Repository in Opalinus Clay Host Rock. Paul Scherrer Institut, Switzerland, 2003.

[3] N.T. Skipper, K. Refson, and J.D.C. McConnell. Computer calculation of water-clay interactions using atomic pair potentials. Clay Minerals, 24(2):411-425, 1989.

[4] N.T. Skipper, F.-R.C. Chang, and G. Sposito. Monte-Carlo simulation of interlayer molecularstructure in swelling clay-minerals, 1. Methodology. Clay Clay Miner., 43(3):285-293, 1995.

[5] D.E. Smith. Molecular computer simulations of the swelling properties and interlayer structure of cesium montmorillonite. Langmuir, 14(20):5959-5967, 1998.

[6] S.H. Park and G. Sposito. Monte-Carlo simulation of total radial distribution functions for interlayer water in Li-, Na- and K-montmorillonite. J. Phys. Chem. B, 104(19):4642-4648, 2000 .

[7] E. Ferrage, B. Lanson, N. Malikova, A. Plancon, B.A. Sakharov, and V.A. Drits. New insights on the distribution of interlayer water in bi-hydrated smectite from X-ray diffraction profile modeling of 001 reflections. Chem. Mater., 17(13):3499-3512, 2005.

[8] E.S. Boek, P.V. Coveney, and N.T. Skipper. Monte Carlo molecular modelling studies of hydrated Li-, Na- and K-smectites: Understanding the role of potassium as a clay swelling inhibitor. $J$. Am. Chem. Soc., 117(50):12608-12617, 1995.

[9] D.A. Young and D.E. Smith. Simulations of clay mineral swelling and hydration: Dependence upon interlayer ion size and charge. J. Phys. Chem. B, 104(39):9163-9170, 2000.

[10] D.E. Smith, Y. Wang, A. Chaturvedi, and H.D. Whitley. Molecular simulations, of the pressure, temperature, and chemical potential dependencies of clay swelling. J. Phys. Chem. B, 110(40):20046-20054, 2006.

[11] X.-D. Liu and X.-C. Lu. A thermodynamic understanding of clay-swelling inhibition by potassium ions. Angew. Chem. Int. Ed., 45(38):6300-6303, 2006.

[12] E.J.M. Hensen, T.J. Tambach, A. Bliek, and B. Smit. Adsorption isotherms of water in Li-, Naand K-montmorillonite by molecular simulation. J. Chem. Phys., 115(7):3322-3329, 2001.

[13] T.J. Tambach, P.G. Bolhuis, E.J.M. Hensen, and B. Smit. Hysteresis in clay swelling induced by hydrogen bonding: Accurate prediction of swelling states. Langmuir, 22(3):1223-1234, 2006.

[14] R. Sutton and G. Sposito. Molecular simulation of interlayer structure and dynamics in 12.4 åCssmectite hydrates. J. Coll. Interf. Sci., 237(2):174-184, 2001.

[15] V. Marry, P. Turq, T. Cartailler, and D. Levesque. Microscopic simulation for structure and dynamics of water and counterions in a monohydrated montmorillonite. J. Chem. Phys., 117(7):3454-3463, 2002. 
[16] N. Malikova, V. Marry, J.-F. Dufrêche, and P. Turq. Na/Cs - montmorillonite: temperature activation of diffusion by simulation. Curr. Opinion in Coll. Int. Sci., 9(1-2):124-127, 2004.

[17] N. Malikova, A. Cadène, V. Marry, E. Dubois, and P. Turq. Diffusion of water in clays on the microscopic scale: modeling and experiments. J. Phys. Chem. B, 110(7):3206-3214, 2006.

[18] R.J.F.L. de Carvalho and N.T. Skipper. Atomistic computer simulation of the clay-fluid interface in colloidal laponite. J. Chem. Phys., 114(8):3727-3733, 2001.

[19] J. A. Greathouse and R. T. Cygan. Molecular dynamics simulation of uranyl(vi) adsorption equilibria onto an external montmorillonite surface. Phys. Chem. Chem. Phys., 7(20):3580$3586,2005$.

[20] J. A. Greathouse and R. T. Cygan. Water structure and aqueous uranyl(vi) adsorption equilibria onto external surfaces of beidellite, montmorillonite, and pyrophyllite: Results from molecular simulations. Env. Sci. Techl., 40(12):3865-3871, 2006.

[21] J. W. Wang, A. G. Kalinichev, and R. J. Kirkpatrick. Molecular modeling of water structure in nano-pores between brucite (001) surfaces. Geochim. Cosmochim. Acta, 68(16):3351-3365, 2004.

[22] R. J. Kirkpatrick, A. G. Kalinichev, and J. W. Wang. Molecular dynamics modelling of hydrated mineral interlayers and surfaces: structure and dynamics. Miner. Mag., 69(3):289-308, 2005.

[23] J. W. Wang, A. G. Kalinichev, R. J. Kirkpatrick, and R. T. Cygan. Structure, energetics and dynamics of water adsorbed on the muscovite (001) surface: A molecular dynamics simulation. J. Phys. Chem. B, 109(33):15893-15905, 2005.

[24] J. W. Wang, A. G. Kalinichev, and R. J. Kirkpatrick. Effects of substrate structure and composition on the structure, dynamics and energetics of water on mineral surfaces: a molecular dynamics modeling study. Geochim. Cosmochim. Acta, 70(3):562-582, 2006.

[25] A. G. Kalinichev, J. W. Wang, and R. J. Kirkpatrick. Molecular dynamics modeling of the structure, dynamics and energetics of mineral/water interfaces: Application to cement materials. Cem. Concr. Res., 37:337-347, 2007.

[26] G. W. Brindley and G. Brown. Crystal Structures of Clay Minerals and their X-ray Identification. Mineralogical Society, London, 1980.

[27] E. Maegdefrau and U. Hoffman. Die Kristallstruktur des Montmorillonits. Z. Kristallogr. Kristallgeom. Kristallphys. Kristallchem., 98:299-323, 1937.

[28] V. Marry and P. Turq. Microscopic simulations of interlayer structure and dynamics in bihydrated heteroionic montmorillonites. J. Phys. Chem. B, 107(8):1832-1839, 2003.

[29] D. Frenkel and B. Smit. Understanding Molecular Simulations, From Algorithms to Applications. Academic Press, 2002.

[30] H.J.C Berendsen, J.R. Grigera, and T.P. Straatsma. The missing term in effective pair potentials. J. Phys. Chem., 91(24):6269-6271, 1987.

[31] S. Koneshan, C. Rasaiah, R.M. Lynden-Bell, and S.H. Lee. Solvent structure, dynamics, and ion mobility in aqueous solutions at $25^{\circ} \mathrm{C}$. J. Phys. Chem. B, 102(21):4193-4204, 1998.

[32] N. Malikova, A. Cadène, V. Marry, E. Dubois, P. Turq, J.-M. Zanotti, and S. Longeville. Diffusion of water in clays - microscopic simulation and neutron scattering. Chem. Phys., 317(2-3):226$235,2005$.

[33] A. Laio, J. VandeVondele, and U. Rothlisberger. D-RESP: Dynamically generated electrostatic potential derived charges from quantum mechanics/molecular mechanics simulations. J. Phys. Chem. B, 106(29):7300-7307, 2002.

[34] B. Kirchner and J. Hutter. Solvent effects on electronic properties from wannier functions in a dimethyl sulfoxide/water mixture. J. Chem. Phys., 121(11):5133-5142, 2004.

[35] B. Rotenberg, V. Marry, R. Vuilleumier, N. Malikova, C. Simon, and P. Turq. Water and ions in clays : Unraveling the interlayer/micropore exchange using molecular dynamics. Geochim. et Cosmochim. Acta, 71:5089-5101, 2007.

[36] W. Smith and T.R. Forester. The DLPOLY2 user manual. CCLRC, Daresbury Laboratory, Daresbury, Warrington, England, 1995. 
[37] J.-P. Ryckaert, G. Ciccotti, and H. J. C. Berendsen. Numerical integration of the cartesian equations of motion of a system with constraints: molecular dynamics of $n$-alkanes. J. Comput. Phys., 23(3):327-341, 1977.

[38] Y. Kim and R.J. Kirkpatrick. ${ }^{23}$ na and ${ }^{133} \mathrm{Cs} \mathrm{nmr}$ study of cation adsorption on mineral surfaces: Local environment, dynamics, and effect of mixed cations. Geochim. Cosmochim. Acta, 61(24):5199-5208, 1997.

[39] B.C. Bostick, M.A. Vairavamurthy, K.G. Karthikeyan, and J. Chorover. Cesium adsorption on clay minerals: An EXAFS spectroscopic investigation. Env. Sci. Tech., 36(12):2670-2676, 2002.

[40] V. Marry, B. Rotenberg, and P. Turq. Structure and dynamics of water at a clay surface from molecular dynamics simulation. Phys. Chem. Chem. Phys., 10(32):4802-4813, 2008.

[41] A. Luzar and D. Chandler. Hydrogen-bond kinetics in liquid water. Nature, 379(6560):55-57, 1996.

[42] A. Luzar and D. Chandler. Effect of environment on hydrogen bond dynamics in liquid water. Phys. Rev. Lett., 76(6):928-931, 1996.

[43] D. Laage and J. T. Hynes. A molecular jump mechanism of water reorientation. Science, 311(5762):832-835, 2006.

[44] S. Kerisit and C.X. Liu. Molecular simulations of water and ion diffusion in nanosized mineral fractures. Env. Sci. Tech., 43(3):777-782, 2009.

[45] S.R.-V. Castrillon, N. Giovambattista, I.A. Aksay, and P.G. Debenedetti. Evolution from surface-influenced to bulk-like dynamics in nanoscopically confined water. J. Phys. Chem. B, 113(23):7973-7976, 2009.

[46] D. van der Spoel, P.J. van Maaren, and H.J.C. Berendsen. A systematic study of water models for molecular simulation: Derivation of water models optimised for use with a reaction field. $J$. Chem. Phys., 108(24):10220-10230, 1998.

[47] K. Krynicki, C.D. Green, and D.W. Sawyer. Pressure and temperature dependence of self-diffusion in water. Faraday Discuss. Chem. Soc., 66:199-208, 1978.

[48] A. Chandra. Effects of ion atmosphere on hydrogen-bond dynamics in aqueous electrolyte solutions effects of ion atmosphere on hydrogen-bond dynamics in aqueous electrolyte solutions. Phys. Rev. Lett., 85(4):768-771, 2000.

[49] A. Luzar. Resolving the hydrogen bond dynamics conundrum. J. Chem. Phys., 113(23):1066310675, 2005.

[50] S. Balasubramanian, S. Pal, and B. Bagchi. Hydrogen-bond dynamics near a micellar surface: Origin of the universal slow relaxation at complex aqueous interfaces. Phys. Rev. Lett., 89(11):115505, 2002.

[51] P. Liu, E. Harder, and B. J. Berne. Hydrogen-bond dynamics in the air-water interface. J. Phys. Chem. B, 109(7):2949-2955, 2005.

[52] P. Liu, E. Harder, and B.J. Berne. On the calculation of diffusion coefficients in confined fluids and interfaces with an application to the liquid-vapor interface of water. J. Phys. Chem. B, 108(21):6595-6602, 2004.

[53] N. Malikova, V. Marry, J.-F. Dufrêche, C. Simon, P. Turq, and E. Giffaut. Temperature effect in a montmorillonite clay at low hydration - microscopic simulation. Mol. Phys., 102(18):1965-1977, 2004.

[54] B. Guillot. A reappraisal of what we have learnt during three decades of computer simulations of water. J. Mol. Liq., 101:219-260, 2002.

[55] B. J. Teppen and D. M. Miller. Hydration energy determines isovalent cation exchange selectivity by clay minerals. Soil Sci. Soc. Am. J., 70(1):31-40, 2006.

[56] L.R. Van Loon and M.A. Glaus. Mechanical compaction of smectite clays increases ion exchange selectivity for cesium. Env. Sci. Tech., 42:1600-1604, 2008.

[57] B. Rotenberg, J.-P. Morel, V. Marry, P. Turq, and N. Morel-Desrosiers. On the driving force of cation exchange in clays : Insights from combined microcalorimetry experiments and molecular simulation. Geochim. et Cosmochim. Acta, 73:4034-4044, 2009. 
[58] V. Marry, J.-F. Dufrêche, M. Jardat, and P. Turq. Equilibrium and electrokinetic phenomena in charged porous media from microscopic and mesoscopic models: electro-osmosis in montmorillonite. Mol. Phys., 101(20):3111-3119, 2003. 